\title{
Autism-Like Behavior in BTBR Mice Is Improved by Electroconvulsive Therapy
}

\author{
Eunice Hagen $^{1}$ - Dana Shprung ${ }^{1} \cdot$ Elena Minakova $^{1} \cdot$ James Washington III $^{1}$. \\ Udaya Kumar $^{1}$ • Don Shin ${ }^{1} \cdot$ Raman Sankar $^{1,2}$ - Andrey Mazarati ${ }^{1}$
}

Published online: 28 April 2015

(C) The American Society for Experimental NeuroTherapeutics, Inc. 2015 treatment of autism, and that its therapeutic effects may be mediated, in part, by central oxytocin signaling.

Keywords Autism $\cdot$ Electroconvulsive therapy $\cdot$ BTBR mice $\cdot$ Oxytocin $\cdot$ Oxytocin receptor blocker

\section{Introduction}

Autism is a considerably debilitating developmental disorder characterized by impairments in social and communications abilities, as well as by restricted and repetitive behaviors [1]. Presently, there are no mechanism-based therapies for autism [2]. This is owing, in part, to the multifactorial nature of the disease and the lack of a single, well-identified signaling pathway underlying autism-associated behavioral and cognitive abnormalities. A wide range of mechanisms causing the disease calls for the exploration of broad, not necessarily pharmacological, interventions. For example, several studies report on the positive effects of a ketogenic diet in patients with autism, as well as in animal models [3, 4].

In our recent studies exploring the relationship between an autism-like phenotype and epilepsy in mice, we found that in animals with pre-existing autism-like impairments (which had been induced by prenatal exposure to viral-like infection), the severity of autism symptoms was alleviated upon the induction of spontaneous recurrent seizures by intrahippocampal kainic acid, and that the extent of behavioral improvement in tests for autism-like behavior positively correlated with the frequency of seizures [5]. This finding, albeit unexpected, was nevertheless congruent with limited clinical evidence suggesting that electroconvulsive therapy (ECT) may have positive effects in patients with autism [6-10]. We surmised that the "antiautistic" effects of seizures observed in our experiments might not be serendipitous but rather have true 
therapeutic potential via the recruitment of relevant and identifiable mechanisms.

While the effects of ECT are likely to be multifaceted and thus impossible to deduce to a single pathway or a molecule, one plausible candidate is oxytocin. Oxytocin is a "social" hormone, and its deficiency has been implicated in mechanisms of autism in both clinical and experimental studies. Clinical studies connected autism to the decreased plasma oxytocin levels, as well as to oxytocin receptor gene polymorphism [11-13]. In the laboratory setting, oxytocin receptor knockout mice presented with autism-like behavioral impairments $[14,15]$. Conversely, oxytocin administration improved social behavioral deficits in several models of autism, such as inbred mouse strains BALB/cByJ and C58/J [16].

With regard to seizures, kainic acid-induced convulsions in rats led to the increased expression of oxytocin in the hypothalamus [17]. Furthermore, ECT in rats was accompanied by an elevated plasma oxytocin level [18]. These observations may explain, at least in part, the mitigation of autism-like behavior in epileptic mice, and therapeutic effects of ECT in autism, which have been alluded to earlier.

In this study we examined whether ECT indeed exerts therapeutic effect in a validated animal model of autism. For this, we chose BTBR $\mathrm{T}+\mathrm{tf} / \mathrm{J}$ mice, which present with a spectrum of behavioral perturbations (e.g., impaired sociability and repetitive behavior), as well as neuropathological abnormalities (e.g., absence of corpus callosum, i.e., deficient long-range connectivity) consistent with autism [19, 20], and are commonly used in autism research. We report that ECT transiently reverses autism-like behavioral abnormalities in BTBR mice, and that therapeutic effects of ECT may be partly regulated by central oxytocin signaling.

\section{Methods}

\section{Animals}

The experiments were performed in male BTBR $\mathrm{T}+\mathrm{tf} / \mathrm{j}$ (hereafter referred to as BTBR) and C57bl/6j mice (The Jackson Laboratory, Bar Harbor, ME, USA), which were 40 days old at the beginning of the study. Animals were kept in a 12-h light (7:00 AM7:00 PM)/dark (7:00 PM-7:00 AM) cycle and had free access to food and water. Experimental procedures were approved by the University of California, Los Angeles, animal research committee and complied with National Institutes of Health and Association for Assessment and Accreditation of Laboratory Animal Care International guidelines and requirements.

\section{Behavioral Tests}

Behavioral testing was performed using the 3-chamber test [21], adapted by our laboratory [22], between 10:00 AM and
1:00 PM. The apparatus was a $60 \times 40$-cm Plexiglas box divided into 3 connected chambers (Noldus, Leesburg, VA, USA). Each of the end compartments contained wired cylindrical enclosures $(11 \mathrm{~cm}$ high, $10 \mathrm{~cm}$ diameter, bar space $1 \mathrm{~cm}$ apart). The test was performed in an isolated sound-proof room, under low-lit conditions. Prior to the testing proper, the test animal was placed inside the chamber and was allowed to habituate for $10 \mathrm{~min}$. Animals' behavior was videotaped and analyzed offline, as described below.

\section{Sociability}

An unfamiliar age-, sex-, and strain-matched mouse (conspecific) was placed inside one of the enclosures, and an unfamiliar object (cube, cylinder or pyramid) inside the other enclosure. The test animal was placed into the central chamber, and was allowed to explore the apparatus for $10 \mathrm{~min}$.

\section{Social Novelty}

Upon completing the sociability phase of the test, the test mouse was removed from the apparatus. The object in the respective enclosure was replaced by a new, unfamiliar mouse; the conspecific in the other enclosure (now-familiar mouse) remained in place. The test mouse was re-introduced into the apparatus and was allowed to explore it for $10 \mathrm{~min}$.

When the sociability and social novelty tests were applied repeatedly to the same animals, for each consecutive test new conspecific mice and new objects were used. Furthermore, according to our experience (unpublished observations), frequent exposure of animals to the apparatus diminishes their overall explorative behavior, and therefore the minimal timing between repeated tests was kept at 2 weeks.

\section{Self-Directed Repetitive Behavior}

Behavior was studied by calculating the cumulative time spent grooming in each chamber during the sociability and social novelty phases of the test. Examining grooming during the sociability tests rather than in isolation, as it is commonly done, allowed us to analyze repetitive behavior in the context of the surroundings.

\section{Analysis of Behavior}

Sociability (first phase) was expressed as the sociability index, which involved calculating time ( $\mathrm{t}$ ) in seconds spent exploring (sniffing) the conspecific and the object using the formula: $\left[\mathrm{t}_{\text {conspecific }} / \mathrm{t}_{\text {conspecific }}+\mathrm{t}_{\text {object }}\right] \times 100-50$. Social novelty (second phase) was expressed as social novelty index and was calculated using a similar formula: $\left[t_{\text {new conspecific }} / t_{\text {new }}\right.$ conspecific $\left.+\mathrm{t}_{\text {old conspecific }}\right] \times 100-50$. On the resulting scale, the sociability and social novelty span from +50 (full preference for the 
conspecific during phase 1 and new conspecific during phase 2) to 0 (social indifference) to -50 (complete avoidance of the conspecific during phase 1 and of new conspecific during phase 2) [22-24]. Repetitive behavior was analyzed by calculating cumulative time spent grooming during each of the phases of the test and in each of the chambers of the apparatus.

\section{ECT}

The ECT protocol followed a commonly described procedure, using a Pulse Generator 57800-001 (Ugo Basile, Comerio, Italy). The supplied auricular clip electrodes were placed on the mouse's ears, and standard electroconvulsive electroshock (ECS) was applied (pulse duration $0.1 \mathrm{~ms}$, ECS duration $1 \mathrm{~s}$, 100 pulses, $25 \mathrm{~mA}$ ) [25, 26]. Three ECT regimens were applied in separate groups of animals (Table 1). 1) Mice received a total of 13 ECSs (twice per day $8 \mathrm{~h}$ apart on days 1-6, and 1 in the morning of day 7). 2) Separate animals were subjected to a procedure of a similar duration (i.e.m 7 days) but of a lower ECS density. These animals received ECS once a day in the morning on days 2-7 (total of 6 ECS); sham procedures (i.e., animals were connected to the pulse generator but no ECS were administered) were delivered twice on day 1 and thereafter were alternated with ECS with 8-h intervals on days 2-7.3) Animals were subjected to 12 consecutive sham procedures given twice a day $8 \mathrm{~h}$ apart, followed by a single ECS on day 7.

A behavioral test was performed $24 \mathrm{~h}$ after the last ECS. The criterion for successful ECS was the presence of generalized tonic convulsion with hindlimb extension lasting for at least $5 \mathrm{~s}$; there were no failures in the occurrence of the convulsions.

\section{Oxytocin Receptor Blocker Treatment}

\section{Experimental Drug and the Delivery System}

We used L-368,899 [(2S)-2-Amino-N-[(1S,2S,4R)-7,7dimethy $1-1-$ [ [ [ $4-(2-$ m e thy 1 ph e ny 1$)-1-$ piperazinyl]sulfonyl]methyl]bicyclo[2.2.1]hept-2-yl]- 4-(methylsulfonyl)butanamide hydrochloride; Tocris, Minneapolis, MN, USA]. The compound is a nonpeptide preferential oxytocin receptor antagonist $\left(\mathrm{IC}_{50}=8.9 \mathrm{nM}\right)$ that displays $>40$-fold selectivity over vasopressin $\mathrm{V}_{1 \mathrm{a}}$ and $\mathrm{V}_{2}$ receptors $\left(\mathrm{IC}_{50}\right.$ values $=370$ and $570 \mathrm{nM}$, respectively) [27].

For the drug delivery, we used an ALZET osmotic pump model 1002 (pumping duration 2 weeks, total volume $100 \mu \mathrm{l}$, nominal pumping rate $0.25 \mu \mathrm{l} / \mathrm{h}$; Durect Corporation, Cupertino, CA, USA). The pump was filled with $490 \mu \mathrm{g} \mathrm{L-368,899}$ dissolved in $100 \mu \mathrm{l}$ of saline (resulting in $35 \mu \mathrm{g}$ delivered over each $24 \mathrm{~h}$ ). The flow moderator of the pump was connected to the cannula of the Brain Infusion Kit 3 via a polypropylene catheter (Durect). The assembly was placed in the sterile tube filled with normal saline and placed inside the incubator at $37^{\circ} \mathrm{C}$ for $48 \mathrm{~h}$ for priming before implantation. Control treatments consisted of delivering either saline in lieu of L-368, 899 , or of administering L-368,899 at $3.5 \mu \mathrm{g}$ /day (i.e., $10 \%$ of the original dose; for this, the pump was filled with $49 \mu \mathrm{g}$ of the compound).

\section{Surgery}

Mice were anesthetized with isoflurane and placed in the mouse adapter (Stoelting, Wood Dale, IL, USA) mounted on the stereotaxic apparatus (David Kopf Instruments, Tujunga, CA, USA). The ALZET pump was placed inside the subcutaneous pocket on the animal's back, and the cannula of the Brain Infusion Kit was stereotaxically implanted into the lateral brain ventricle (from Bregma: posterior $0.22 \mathrm{~mm}$, left $1.0 \mathrm{~mm}$, ventral $2.5 \mathrm{~mm}$ [28]). The cannula was fixed to the skull by dental acrylic.

\section{Experiments}

During first 5 days after surgery, the drug was delivered without ECS. Starting from day 6 after surgery, animals underwent ECT as described above. A behavioral test was performed $24 \mathrm{~h}$ after the last ECS (which coincided with the end of the drug delivery).

Table 1 Electroconvulsive therapy (ECT) regimens

\begin{tabular}{|c|c|c|c|c|c|c|c|c|c|c|c|c|c|c|}
\hline \multirow{2}{*}{$\begin{array}{l}\text { ECT day } \\
\text { Time }\end{array}$} & \multicolumn{2}{|l|}{1} & \multicolumn{2}{|l|}{2} & \multicolumn{2}{|l|}{3} & \multicolumn{2}{|l|}{4} & \multicolumn{2}{|l|}{5} & \multicolumn{2}{|l|}{6} & \multicolumn{2}{|l|}{7} \\
\hline & $\mathrm{AM}$ & PM & AM & PM & $\mathrm{AM}$ & PM & $\mathrm{AM}$ & PM & $\mathrm{AM}$ & PM & $\mathrm{AM}$ & PM & $\mathrm{AM}$ & PM \\
\hline $13 \mathrm{ECS}$ & ECS & ECS & ECS & ECS & ECS & ECS & ECS & ECS & ECS & ECS & ECS & ECS & ECS & None \\
\hline $6 \mathrm{ECS}$ & Sham & Sham & ECS & Sham & ECS & Sham & ECS & Sham & ECS & Sham & ECS & Sham & ECS & None \\
\hline $1 \mathrm{ECS}$ & Sham & Sham & Sham & Sham & Sham & Sham & Sham & Sham & Sham & Sham & Sham & Sham & ECS & None \\
\hline
\end{tabular}

Shown are 3 ECT regimens used in the study. For each regimen, the total duration of the procedure was 7 days. Time between AM and PM procedures was $8 \mathrm{~h}$. Behavioral test was performed $24 \mathrm{~h}$ after the last electroconvulsive electroshock (ECS)

Sham $=$ animals were connected to the pulse generator but no ECS was delivered 
After the completion of the experiments, animals were euthanized by pentobarbital $\mathrm{Na}$, brains were removed, kept on dry ice, and placed in brain matrix. Coronal sections were cut at the level of the cannula, to verify its placement in the lateral brain ventricle. The proper cannula position was confirmed for all mice.

\section{Data Analysis}

Data were analyzed in a "blinded fashion" using Prism 6 software (GraphPad, San Diego, CA, USA). Parametric tests were used, as the samples represented interval scales, and passed D'Agostino-Pearson omnibus normality test. One-way analysis of variance, repeated measures analysis of variance, and paired and unpaired Student's $t$ tests were used as appropriate. Statistical tests and sample numbers for each experiment are indicated in the figure legends.

\section{Results}

\section{Comparison of Behavior Between BTBR and C57bl/6j Mice}

In order to characterize autism-like behavior in BTBR animals, before proceeding with the experiments proper we compared their performance with that of $\mathrm{C} 57 \mathrm{bl} / 6 \mathrm{j}$ mice, which are genetically, behaviorally, and anatomically normal.

During the habituation phase of the test, animals had no bias towards either of the end chambers (not shown). During the sociability phase, both BTBR and C57bl/6j mice directly engaged more with a conspecific than with the object; however, $\mathrm{C} 57 \mathrm{bl} / 6 \mathrm{j}$ mice spent a significantly longer time exploring the conspecific versus the object than BTBR mice (Fig. 1a). During the social novelty phase of the test, BTBR mice showed slight-to-no preference towards either of the 2 conspecifics, while $\mathrm{C} 57 \mathrm{bl} / 6 \mathrm{j}$ mice engaged significantly more with a new conspecific than the old one (Fig. 1a). It could be argued that poor social novelty in BTBR mice was due to the insufficient attention paid to a conspecific during the sociability phase (and, consequently, perception of both old and new conspecifics as unfamiliar). However, this was not the case, as the durations of exploring a conspecific during the sociability phase were similar between BTBR and C57bl/6j animals $(49.14 \pm 12.04 \mathrm{~s}$ and $47.00 \pm 6.72 \mathrm{~s}$, respectively; $p>0.05$, Student's $t$ test).

At the same time, during each of the 2 phases of the sociability test, both BTBR and C57bl/6j mice spent a statistically similar amount of time in each of the terminal compartments, with the time spent in the central compartment not exceeding $50 \mathrm{~s}$ (data not shown). As in our hands no differences between the 2 strains were observed in this regard, a mere presence in a target compartment did not appear to be a sensitive enough
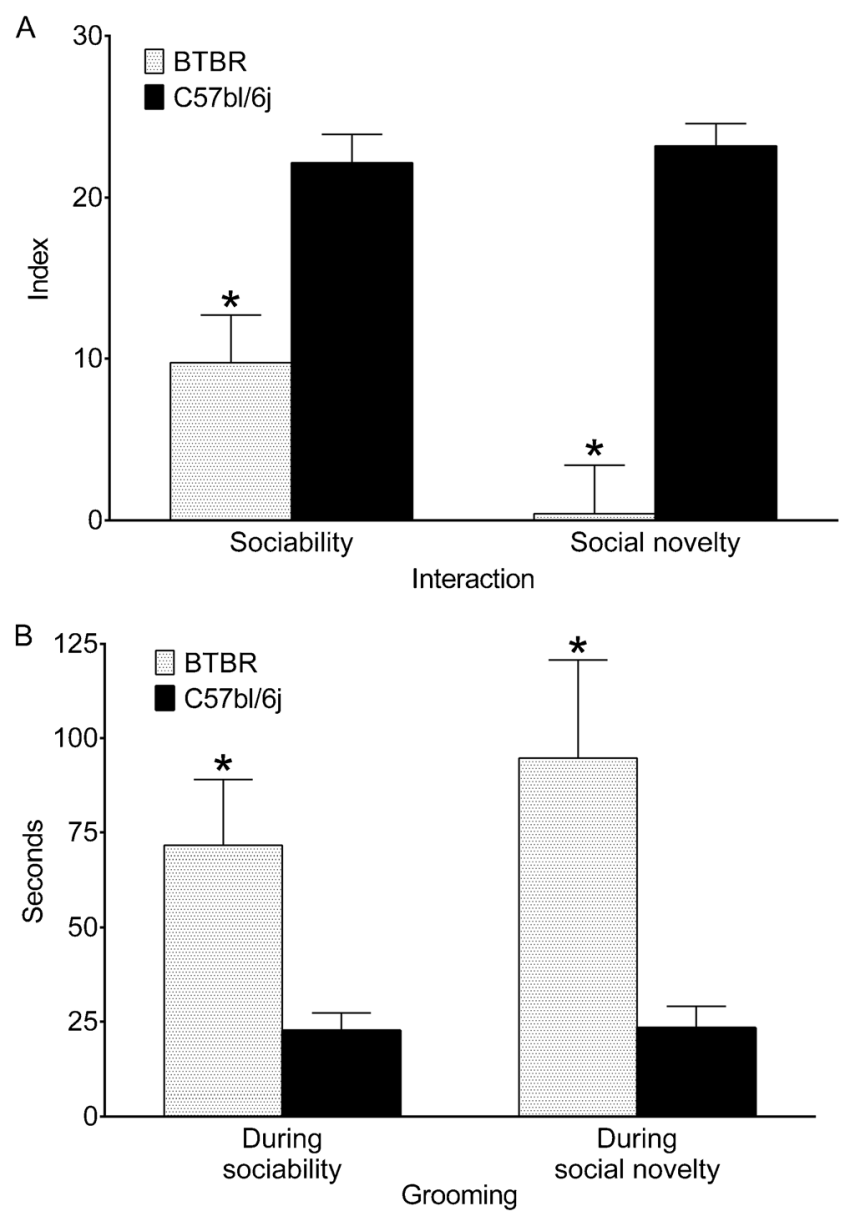

Fig. 1 Comparison of behavior between BTBR and genetically normal C57bl/6j mice. (a) BTBR mice showed lower levels of direct social engagement (sniffing) both during sociability and social novelty phases of the 3-chamber test compared with $\mathrm{C} 57 \mathrm{bl} / 6 \mathrm{j}$ mice. (b) BTBR mice spent more time grooming during each of the phases of the 3-chamber test than their $\mathrm{C} 57 \mathrm{bl} / 6 \mathrm{j}$ counterparts. Sample sizes: BTBR, $n=7$; C57Bl, $n=6$. Data are presented as mean \pm SEM. ${ }^{*} p<0.05 v s$ C57Bl ( $t$ test)

indicator of autism-like behavior. Therefore, in further experiments we only analyzed direct engagement of the test mouse with the conspecific versus the object and a new versus familiar conspecific during sociability and social novelty tests, respectively.

During both phases of the 3-chamber test, BTBR animals spent significantly more time grooming than $\mathrm{C} 57 \mathrm{bl} / 6 \mathrm{j}$ mice. For each strain, the distribution of grooming between the sociability and the social novelty phases was similar (Fig. 1b). However, time spent grooming in a specific chamber during each of the phases differed between the two strains (discussed under "Structure of Repetitive Behavior and Effects of Treatments").

\section{Effects of ECT in BTBR Mice}

These experiments were performed in BTBR mice described above (i.e., in experiments comparing them with $\mathrm{C} 57 \mathrm{bl} / 6 \mathrm{j}$ 
Fig. 2 Effects of electroconvulsive therapy (ECT) on behavioral deficits in BTBR mice. (a) Twenty-four $h$ after the end of ECT, both sociability and social novelty significantly increased compared with baseline (ECT I +1 day $v s$ before ECT I). Two weeks after ECT (ECT I +2 weeks), its effects diminished but persisted so that both sociability and social novelty were statistically similar to respective parameters observed before ECT and $24 \mathrm{~h}$ after ECT. The administration of the second ECT (ECT II + 1 day) produced effects similar to those observed after the first ECT. The effects of ECT fully disappeared 1 month after the treatment (ECT II + 1 month). (b) ECT effectively reduced cumulative duration of grooming during the 3-chamber test. The effects were fully congruent with those on sociability and social novelty (i.e., full reversal $24 \mathrm{~h}$ after ECT, partial diminution at 2 weeks, and complete disappearance at 1 month) Data are presented as mean $\pm \operatorname{SEM}(n=7) * p<0.05 v s$ "Before ECT I". Repeated measures analysis of variance plus Tukey's multiple comparison test for each test. $F(1.981,11.89)=12.6$ for sociability; $F(2.161,12.96)=13$ for social novelty; $F(2.191,13.15)=10.92$ for grooming during sociability phase; $F(1.705,10.23)=10.1$ for grooming during social novelty phase. (c) Effects of single electroconvulsive electroshock (ECS) and 6 ECS on behavior in BTBR mice. Treatments consistent of 12 consecutive sham ECS followed by 1 ECS (12 sham +1 ECS; $n=5)$ and of 7 sham ECS administered intermittently with ECS ( 7 sham +6 ECS; $n=5)$. Neither of the protocols had statistically significant effects on the animals' behavior ( $p>0.05$, paired $t$ test). Data are presented as mean \pm SEM per cent of parameters before respective treatments (baseline); for sociability and social novelty, data are normalized on the -50 to +50 scale by adding 50 to absolute index values (so as to avoid negative per cent). For statistical analysis, absolute values were compared

strain). After the examination of baseline behavior [Fig. 1 and $2 \mathrm{a}$ and $\mathrm{b}$ ("Before ECT I")], the animals were subjected to 13 consecutive ECS, as described in the "Methods". Behavioral testing $24 \mathrm{~h}$ after the last ECS revealed full reversal of impairments of both sociability and social novelty, whereby both indices were significantly higher than the ones prior to the ECT [Fig. 2a ("Before ECT I and ECT I+ 1 day")], and were in the ranges observed in $\mathrm{C} 57 \mathrm{bl} / 6 \mathrm{j}$ mice. The duration of grooming also significantly decreased during both phases of the test [Fig. 2b ("Before ECT I and ECT I + 1 day")], and was also in the range observed in $\mathrm{C} 57 \mathrm{bl} / 6 \mathrm{j}$ mice.

During the examination 2 weeks after the ECT, animals' behavior during sociability, social novelty tests, and grooming duration showed signs of returning to pre-ECT levels; all examined parameters were statistically similar to the ones both before ECT, and 1 day after ECT [Fig. 2a and b ("ECT I + 2 weeks")].

When the second ECT was administered, animals' behavior once again significantly improved to the levels similar to those observed after the first ECT [Fig. 2a and b ("ECT II + 1 day" compared with "ECT I +1 day")].

Therapeutic effects of the ECT on both sociability and grooming completely disappeared 1 month after the treatment [Fig. 2a and b ("ECT II + 1 month")].

\section{Effects of Single and 6 ECS}

Neither 12 sham procedures followed by single or 6 ECS alternated with 7 sham procedures had any statistically
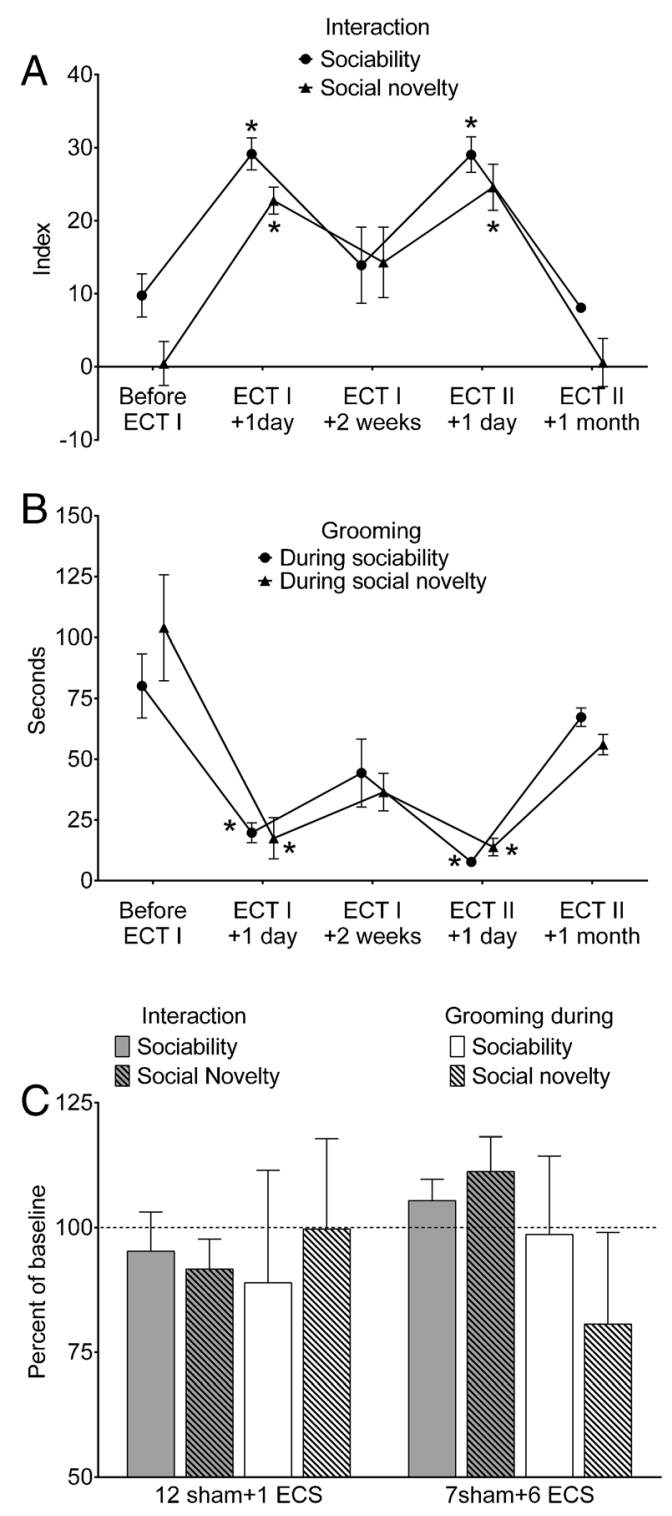

significant effects on animals' behavior in sociability, social novelty, and grooming assays (Fig. 2c).

\section{Effects of Oxytocin Receptor Antagonist}

After L-368,899 treatment combined with 13 ECSs, sociability in BTBR mice remained at the same levels as before the procedure, meaning that the blockade of oxytocin receptors completely abolished the therapeutic effects of ECT (Fig. 3a, "Sociability"). At the same time, social novelty was still improved by ECT, even in the presence of oxytocin receptor blocker, although it did not reach levels observed when ECT was administered during saline infusion in lieu of L-368,899 (Fig. 3a, "Social novelty"). Therefore, blockade of oxytocin receptors did not abolish, but partially antagonized, the therapeutic effect of ECT. Administration of L-368,899 did not affect ECT-induced reduction of grooming behavior, which 
Fig. 3 Effects of co-administration of electroconvulsive therapy (ECT) with oxytocin receptor blocker (2S)-2-Amino-N-[(1S,2S,4R)-7,7-dimethyl-1-[[[4(2-methylphenyl)-1-piperazinyl]sulfonyl]methyl]bicyclo[2.2.1]hept-2-yl]-4(methylsulfonyl)butanamide hydrochloride (L-368,899) on behavioral deficits in BTBR mice. (a) Treatment with an oxytocin receptor blocker (OxyR blocker), L-368,899, prevented ECT-induced improvement of sociability. Under OxyR blocker ECT still improved social novelty; however, this effect was not as pronounced as when ECT was administered in the presence of saline in lieu of L-368-899 (After Saline/ECT vs After OxyR blocker ECT; $p<0.05$, Student's $t$ test). (b) Even in the presence of OxyR blocker, ECT fully reversed increased grooming behavior in BTBR mice. OxyR blocker $n=7$; saline $n=5$. Data are presented as mean \pm SEM. $* p<0.05$ "After OxyR blocker/ECT" vs "Before OxyR blocker/ECT; ${ }^{\dagger} p<0.05$ "After Saline/ECT" vs "Before Saline/ECT" (paired $t$ tests). (c) The administration of OxyR blocker at $3.5 \mu \mathrm{g} /$ day (i.e., $10 \%$ of the effective dose) had no effects on therapeutic effects of ECT $(n=5)$. When administered to C57Bl mice $(n=6)$, OxyR blocker showed no proper effects on behavior. $* p<0.05$ when comparing data after treatments with data before treatments (paired $t$ test). Data are presented as mean \pm SEM per cent of parameters before respective treatments (baseline); for sociability and social novelty, data are normalized on the -50 to +50 scale by adding 50 to absolute index values (so as to avoid negative per cent). For statistical analysis, absolute values were compared

not only significantly improved compared with pre-ECT values, but was in a range observed when ECT was administered during intracerebral saline infusion (Fig. 3b).

Administration of L-368,899 at $10 \%$ of the effective dose (i.e., at $3.5 \mu \mathrm{g} / \mathrm{day}$ ) to BTBR mice did not modify therapeutic effects of ECT on any of the 3 behavioral parameters (Fig. 3c, first group of 4 bars shows significant improvement of all parameters).

In order to clarify whether L-368,899 had proper detrimental effects on behavior in the applied dose, we administered the compound in $\mathrm{C} 57 \mathrm{bl} / 6 \mathrm{j}$ mice. We chose to limit this experiment to $\mathrm{C} 57 \mathrm{bl} / \mathrm{j} 6$ animals because BTBR mice are already impaired, and considering the numerical values of their behavioral parameters, further deterioration of behavior might be impossible to reveal. C57bl/j6 mice did not undergo ECT but were subjected to 13 sham procedures. When tested in C57b1/6j mice in the absence of ECT, L-368,899 at the applied dose of $35 \mu \mathrm{g} /$ day showed no proper effects on any of the analyzed behaviors (Fig. 3c, second group of 4 bars).

\section{Structure of Repetitive Behavior and Effects of Treatments}

BTBR and C57bl/6j mice showed different patterns of distribution of grooming behavior between the end chambers during both sociability and social novelty phases. During the sociability phase, BTBR mice spent more time grooming in the chamber with a conspecific, while $\mathrm{C} 57 \mathrm{bl} / 6 \mathrm{j}$ mice preferred grooming in a chamber with an object (Fig. 4, first 2 bars in the left group). During the social novelty phase, BTBR mice groomed more in the presence of a new conspecific as compared with the familiar one, while, conversely, C57bl/6j mice spent more time grooming in the chamber with the familiar
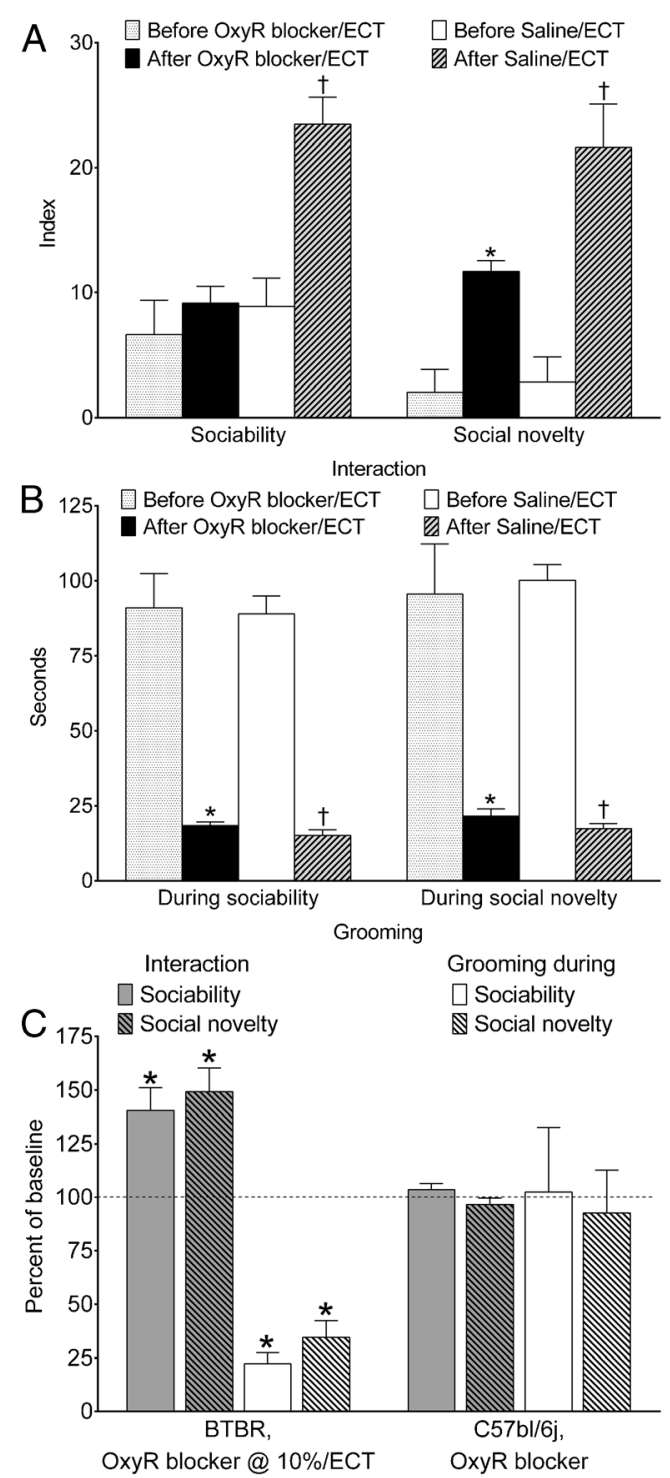

conspecific (Fig. 4, first 2 bars in the right group). Both BTBR and $\mathrm{C} 57 \mathrm{bl} / 6 \mathrm{j}$ mice spent little time grooming in the center chamber $(0-10 \mathrm{~s})$, with no differences between the 2 strains (not shown).

After ECT was administered to BTBR animals the duration of grooming was not only reduced, but its structure also started to resemble of that in $\mathrm{C} 57 \mathrm{bl} / 6 \mathrm{j}$ mice [i.e., increased grooming in the presence of the object during the sociability phase, and in the presence of the familiar conspecific, during the social novelty phase (Fig. 4, BTBR/ECT)]. As for effects of L-368,899, although the blockade of oxytocin receptors did not affect ECT-induced reduction of grooming per se, it did reverse the distribution of grooming between the 2 chambers to the pattern observed in the untreated BTBR animals [i.e., preferential grooming in the chamber with the conspecific vs the object and with new conspecific $v s$ the familiar one during 


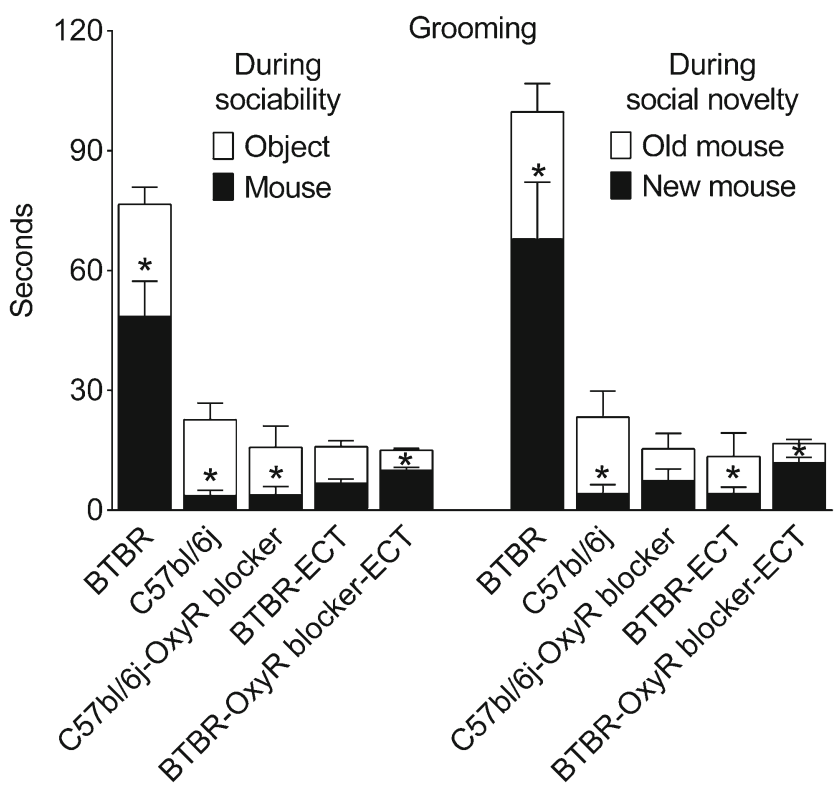

Fig. 4 Structure of repetitive behavior and effects of treatments. Grooming (in seconds) is shown in each of the end chambers for the sociability and social novelty phases of the test. BTBR mice $(n=7)$ spent more time grooming in the presence of a conspecific during sociability and in the presence of a new conspecific during social novelty phases. In C57B1 mice $(n=6)$, the distribution of grooming was the opposite of that in BTBR. Electroconvulsive therapy (ECT) applied to BTBR $(n=7)$ not only reduced total grooming duration, but also changed its distribution in the direction similar to that in C57B1 mice. Under the administration of an oxytocin receptor blocker (OxyR blocker) (2S)-2Amino-N-[(1S,2S,4R)-7,7-dimethyl-1-[[[4-(2-methylphenyl)-1piperazinyl]sulfonyl]methyl]bicyclo[2.2.1]hept-2-yl]-4(methylsulfonyl)butanamide hydrochloride $(n=7), \mathrm{ECT}$, while shortening the duration of grooming, failed to inverse the distribution of repetitive behavior as it did when applied alone. Data are presented as mean $\pm \mathrm{SEM}$. ${ }^{*} p<0.05$ object $v s$ mouse for sociability and old mouse $v s$ new mouse for social novelty $(t$ test $)$

the sociability and social novelty phases respectively; Fig. 4 $(B T B R / L-368,899 / E C T)]$.

\section{Discussion}

Our studies show that 1) in BTBR mice, when the animals are tested $24 \mathrm{~h}$ after the end of the treatment, ECT reverses such behavioral symptoms of autism as impaired sociability, impaired social novelty, and repetitive behavior; 2) the effect of ECT lasts for about 2 weeks upon the cessation of the treatment but can be re-induced by the second ECT series; 3 ) the therapeutic effect of ECT may, in part, involve plastic changes in the brain, as it requires repeated ECSs; 4) with the used dose of a preferential oxytocin receptor blocker it appears that therapeutic effects of ECT may be mediated by central oxytocin receptors in a complex, behavior-dependent fashion: improvement of sociability depends solely on oxytocin receptors, while the improvement of social novelty relies only partly on oxytocin receptors, and the improvement of repetitive behavior does not involve oxytocin receptors.

ECT represents an effective treatment of pharmacoresistant cases of depression [29-31], bipolar disorder [32], and schizophrenia $[33,34]$. Mechanisms underlying the therapeutic effects of ECT remain largely elusive, with 2 major hypotheses dominating: the anticonvulsant, whereby the effects are attributed to the increase of seizure threshold and decrease of seizure duration observed during the course of ECT [35], and the neurotrophic, which brings in the induction of several neurotrophic factors (e.g., brain-derived neurotrophic factor, vascular endothelial growth factor), transcription factors (e.g., cfos), and neurogenesis as explanations [36, 37].

ECT has not been extensively used for the treatment of autism and no controlled clinical trials have been performed. Several studies report on beneficial effects of ECT in patients with autism. Most of these studies are case reports, showing improvement of catatonia and self-injurious behavior [6-10], although beneficial effects on intellectual and adaptive functioning have also been observed [38]. No theoretical basis for the potential therapeutic effect of ECT in autism has been discussed. Certainly, social, psychological, and professional barriers exist, which hinder wider use of ECT for the treatment of psychiatric disorders [39, 40]. From this standpoint, preclinical studies that could show an unambiguous benefit of ECT in validated animal models of autism are useful, as they can build the framework for further mechanism-oriented research and, if successful, validate the use of ECT in patients with severe autism symptoms. To our knowledge, ours is the first report showing that ECT can, indeed, be effective in a commonly used experimental model of autism.

In BTBR mice, ECT improved impairments in all of the 3 analyzed behaviors: sociability, social novelty, and repetitive behavior. The effectiveness of ECT in BTBR mice is particularly remarkable considering that these animals have profound and irreversible deficiency in interhemispheric connectivity due to the missing corpus callosum and underdeveloped hippocampal commissure [41, 42], which is congruent with compromised long-range connectivity in autism [43-45]. However, it should be noted that the lack of corpus callosum alone cannot explain the presence of autism-like behavior; indeed, postnatal mechanical lesion to the corpus callosum in $\mathrm{LP} / \mathrm{J}$ mice (which are genetically close to BTBR mice but show no autism-like behavioral abnormalities) failed to produce autism-like impairments [46]. Concurrently with the compromised long-range connectivity, BTBR mice exhibit aberrant intrahemispheric connectivity as identified by diffusion tensor imaging [20], which is another widely acknowledged attribute of the autistic brain [47]. With this regard, ECT was shown to reduce local connectivity in prefrontal cortex [29], an effect which, if experimentally confirmed, could explain, at least in part, its therapeutic effect in BTBR mice. 
At the same time, it is highly plausible that mechanisms of beneficial effects of ECT in autism (much like in the discussed psychiatric disorders) cannot be deduced to a single pathway or a molecule but rather involve a variety of targets. Furthermore, 2 types of effects can be contemplated: one is restoring a specific dysfunction and another is an overcompensation of otherwise unaffected pathways.

Among potential candidate targets, in this proof-ofprinciple study we decided to focus on oxytocin. By employing a preferential oxytocin receptor blocker (but see discussion of its limitations below), we pursued obtaining an initial signal on potential role of this hormone in the therapeutic effect of ECT, which, depending on outcomes, would have either prompted or discouraged further studies.

A positive regulatory role of oxytocin in social behaviors has been widely accepted. Oxytocin promotes social behavior, while its deficiency (e.g., oxytocin or oxytocin receptor gene polymorphism) has been implicated in mechanisms of autism [11-13]. Oxytocin and oxytocin receptor knockout mice present with autism-like abnormalities [14, 15], whereas exogenously administered oxytocin improves autism-like behavior in mice of the BALB/cByJ and C58/J strains [16]. Treatment of Magel-2 deficient mice (which show autism-like impairments over the lifespan) with oxytocin during early postnatal age rescued their social behavior phenotype in the adulthood [48].

Furthermore, several studies showed that ECT increases plasma oxytocin levels in patients with depression [49-51], as well as in rats [18], which together with the prosocial effects of the hormone supports its potential mediation of therapeutic effects of ECT.

It should be noted that BTBR mice are characterized by increased, rather than suppressed, oxytocin expression in paraventricular hypothalamic nucleus, and by the increased expression of postsynaptic oxytocin receptors [52]. These changes may represent overcompensatory mechanisms, which remain insufficient to overcome other dysfunctions liable to the autism-like phenotype in BTBR mice. Furthermore, long-term administration of intranasal oxytocin reportedly failed to improve behavior in BTBR mice [53].

Nevertheless, in our experiments central delivery of a preferential oxytocin receptor blocker, L-368,899, abolished ECTinduced improvement of sociability, and significantly mitigated the improvement of social novelty. Assuming preferential blockade of oxytocin receptors by L-368,899 in our protocol, it is conceivable that ECT induced further overexpression of oxytocin and/or oxytocin receptor, which then became sufficient to play a compensatory role and thus normalize the animals' behavior. Alternatively, there is a possibility that immediate and prolonged effects of ECT (e.g., $24 \mathrm{~h} v s$ 1-2 weeks) have different underlying mechanisms. In our experiments we only tested L-368,899/ECT animals $24 \mathrm{~h}$ after the last ECS and did not re-test them later for technical reasons: on the one hand, at 2 weeks, the effects of ECT proper begin to dissipate, which would substantially complicate the interpretation of the outcomes of L-368,899 treatment; on the other hand, as mentioned in the "Methods", we were avoiding repeating the test more frequently that every 2 weeks, as this diminishes animals' explorative activity and thus may skew the outcome of the employed assays. Further analysis of oxytocin and oxytocin receptor expression in the brain may clarify the involvement of this hormone in therapeutic effect of ECT.

While the improvement of social deficits by ECT were abolished/mitigated by L-368,899, the drug did not affect ECT-induced shortening of grooming time, thus suggesting that oxytocin did not mediate the effects of ECT on repetitive behavior in the examined system. In clinical studies, oxytocin treatment mitigated repetitive behavior in male patients with autism [54]; however, no correlation was found between plasma oxytocin levels and repetitive behavior in children with autism [55].

ECT normalized the social aspect of grooming behavior in $\mathrm{BTBR}$ mice vis-à-vis $\mathrm{C} 57 \mathrm{bl} / \mathrm{j} 6$ counterparts. An explanation that we can offer for the observed distribution of grooming behavior is that $\mathrm{C} 57 \mathrm{bl} / \mathrm{j} 6$ animals, in which grooming, presumably, is a part of a predominantly normal behavioral repertoire, prefer grooming while being away from an object that they perceive to be engaging (i.e., conspecific and novel conspecific during the sociability and social novelty phases, respectively). In BTBR mice, preferential grooming in the proximity of a conspecific/novel conspecific may instead be a manifestation of social anxiety [56]. With such an explanation in mind, the realignment of grooming by ECT and blockade of this realignment by L-368,899 is congruent with the observed effects on social approach during the sociability test proper.

While L-368,899 shows a reasonably high preference towards oxytocin receptors versus vasopressin receptors (approximately 40-fold), it also binds to $\mathrm{V}_{1 \mathrm{a}}$ and $\mathrm{V}_{2}$ vasopressin receptors [27]. It is difficult, if impossible, to choose a dose that would not affect vasopressin receptors upon in vivo drug delivery. We approximated the dose of the drug based on prior studies [57], and in our experiments at least one-tenth of the effective dose did not modify the effects of ECT at all. Still, the involvement of vasopressin receptors cannot be discounted, particularly considering that vasopressin has been implicated in regulating social behaviors and mechanisms of autism [58, 59]. A more definitive answer could be obtained from the examination of autism in oxytocin and/or oxytocin knockout mice. At the same time, the presented pharmacological analysis (albeit limited) had an advantage as it allowed us to dissociate effects of L-368,899 on the 3 behaviors. While, admittedly, our experiments do not provide conclusive evidence as to the mediation of effects of ECT by oxytocin receptors, the obtained data represent a positive first step 
towards dissecting mechanisms that may underlie effects of ECT in autism and at least warrant further examination of the role of oxytocin signaling.

Other possible mechanisms of action of ECT cannot be excluded. For example, imaging studies may reveal normalization of functional aberrations in intrahemispheric connectivity, which, as mentioned earlier, is perturbed in BTBR mice [47].

Overall, it is highly plausible that in different animal models, ECT would modify autism-like behaviors in a different fashion and through different mechanisms. Therefore, future experimental studies should include the examination of the effects of ECT in various models of autism, and in each of those models, where it is proven effective, possible underlying mechanisms should be analyzed. Such studies would help to build a therapeutic profile of ECT depending on concrete cases and causes of autism.

In conclusion, our experiments provide evidence that ECT may effectively improve autism-like behavior in a validated mouse model. The involvement of oxytocin signaling in therapeutic effects of ECT requires further examination; however, based on the observed initial effects it appears to be a promising target.

Acknowledgements This work was supported by National Institutes of Health research grants R01 NS065783 and R21 NS089396 to A.M., and by a research and training grant from the Division of Neonatology, Department of Pediatrics, University of California, Los Angeles, to E.H. and E.M.

Required Author Forms Disclosure forms provided by the authors are available with the online version of this article.

\section{References}

1. Diagnostic and Statistical Manual of Mental Disorders V. American Psychiatric Association, Washington, DC, 2013.

2. Chadman KK, Guariglia SR, Yoo JH. New directions in the treatment of autism spectrum disorders from animal model research. Expert Opin Drug Discov 2012;7:407-416.

3. Ruskin DN, Svedova J, Cote JL, et al. Ketogenic diet improves core symptoms of autism in BTBR mice. PLoS One 2013;8:e65021.

4. Napoli E, Duenas N, Giulivi C. Potential therapeutic use of the ketogenic diet in autism spectrum disorders. Front Pediatr 2014;2: 69.

5. Mazarati A, Washington III JA, Sankar R, Shin D, Kumar U. Interaction between recurrent seizures and autism-like behavior in a mouse model of maternal immune activation. American Epilepsy Society Annual Meeting 2014: Abstract \# 1.056.

6. Mazzone L, Postorino V, Valeri G, Vicari S. Catatonia in patients with autism: prevalence and management. CNS Drugs 2014;28: 205-215.

7. Wachtel LE, Jaffe R, Kellner CH. Electroconvulsive therapy for psychotropic-refractory bipolar affective disorder and severe selfinjury and aggression in an 11-year-old autistic boy. Eur Child Adolesc Psychiatry 2011;20:147-152.
8. Zaw FK, Bates GD, Murali V, Bentham P. Catatonia, autism, and ECT. Dev Med Child Neurol 1999;41:843-845.

9. Dhossche DM, Stanfill S. Could ECT be effective in autism? Med Hypotheses 2004;63:371-376.

10. Wachtel LE, Dhossche DM, Kellner CH. When is electroconvulsive therapy appropriate for children and adolescents? Med Hypotheses 2011;76:395-399.

11. Nyffeler J, Walitza S, Bobrowski E, Gundelfinger R, Grunblatt E. Association study in siblings and case-controls of serotonin- and oxytocin-related genes with high functioning autism. J Mol Psychiatry 2014;2:1.

12. Parker KJ, Garner JP, Libove RA, et al. Plasma oxytocin concentrations and OXTR polymorphisms predict social impairments in children with and without autism spectrum disorder. Proc Natl Acad Sci U S A 2014;111:12258-12263.

13. Striepens N, Kendrick KM, Maier W, Hurlemann R. Prosocial effects of oxytocin and clinical evidence for its therapeutic potential. Front Neuroendocrinol 2011;32:426-450.

14. Modi ME, Young LJ. The oxytocin system in drug discovery for autism: animal models and novel therapeutic strategies. Horm Behav 2012;61:340-350.

15. Pobbe RL, Pearson BL, Blanchard DC, Blanchard RJ. Oxytocin receptor and Mecp2 308/Y knockout mice exhibit altered expression of autism-related social behaviors. Physiol Behav 2012;107: 641-648.

16. Teng BL, Nonneman RJ, Agster KL, et al. Prosocial effects of oxytocin in two mouse models of autism spectrum disorders. Neuropharmacology 2013;72:187-196.

17. Sun Q, Pretel S, Applegate CD, Piekut DT. Oxytocin and vasopres$\sin$ mRNA expression in rat hypothalamus following kainic acidinduced seizures. Neuroscience 1996;71:543-554.

18. Gur E, Dremencov E, Garcia F, et al. Functional effects of chronic electroconvulsive shock on serotonergic 5-HT(1A) and 5-HT(1B) receptor activity in rat hippocampus and hypothalamus. Brain Res 2002;952:52-60.

19. McFarlane HG, Kusek GK, Yang M, et al. Autism-like behavioral phenotypes in BTBR T + tf/J mice. Genes Brain Behav 2008;7:152163.

20. Sforazzini F, Bertero A, Dodero L, et al. Altered functional connectivity networks in acallosal and socially impaired BTBR mice. Brain Struct Funct 2014.

21. Crawley JN. Chapter 9. Social Behaviors. In: Craige CP (ed.) What's wrong with my mousee? Wiley Interscience, Hoboken, NJ, 2007, pp. 206-224.

22. Pineda E, Shin D, You SJ, et al. Maternal immune activation promotes hippocampal kindling epileptogenesis in mice. Ann Neurol 2013;74:11-19.

23. Smith SE, Li J, Garbett K, Mirnics K, Patterson PH. Maternal immune activation alters fetal brain development through interleukin-6. J Neurosci 2007;27:10695-10702.

24. Malkova NV, Yu CZ, Hsiao EY, Moore MJ, Patterson PH. Maternal immune activation yields offspring displaying mouse versions of the three core symptoms of autism. Brain Behav Immun 2012;26: 607-616.

25. Sakaida M, Sukeno M, Imoto Y, et al. Electroconvulsive seizureinduced changes in gene expression in the mouse hypothalamic paraventricular nucleus. J Psychopharmacol 2013;27:1058-1069.

26. Weber T, Baier V, Lentz K, et al. Genetic fate mapping of type-1 stem cell-dependent increase in newborn hippocampal neurons after electroconvulsive seizures. Hippocampus 2013;23:1321-1330.

27. Williams PD, Anderson PS, Ball RG, et al. 1-((7,7-Dimethyl-2(S)(2(S)-amino-4-(methylsulfonyl)butyramido)bicyclo [2.2.1]-heptan1(S)-yl)methyl)sulfonyl)-4-(2-methylphenyl)piperaz ine (L-368, 899): an orally bioavailable, non-peptide oxytocin antagonist with potential utility for managing preterm labor. J Med Chem 1994;37: $565-571$. 
28. Paxinos G, Franklin BJ. The mouse brain in stereotaxic coordinates. Academic Press, San Diego, CA, 2001.

29. Abbott CC, Gallegos P, Rediske N, Lemke NT, Quinn DK. A review of longitudinal electroconvulsive therapy: neuroimaging investigations. J Geriatr Psychiatry Neurol 2014;27:33-46.

30. Spaans HP, Kho KH, Verwijk E, Kok RM, Stek ML. Efficacy of ultrabrief pulse electroconvulsive therapy for depression: a systematic review. J Affect Disord 2013;150:720-726.

31. Kellner CH, Greenberg RM, Murrough JW, et al. ECT in treatmentresistant depression. Am J Psychiatry 2012;169:1238-1244.

32. Versiani M, Cheniaux E, Landeira-Fernandez J. Efficacy and safety of electroconvulsive therapy in the treatment of bipolar disorder: a systematic review. J ECT 2011;27:153-164.

33. Pompili M, Lester D, Dominici G, et al. Indications for electroconvulsive treatment in schizophrenia: a systematic review. Schizophr Res 2013;146:1-9.

34. Zervas IM, Theleritis C, Soldatos CR. Using ECT in schizophrenia: a review from a clinical perspective. World J Biol Psychiatry 2012;13:96-105

35. Sackeim HA. The anticonvulsant hypothesis of the mechanisms of action of ECT: current status. J ECT 1999;15:5-26.

36. Segi-Nishida E. Exploration of new molecular mechanisms for antidepressant actions of electroconvulsive seizure. Biol Pharm Bull 2011;34:939-944.

37. Rotheneichner P, Lange S, O'Sullivan A, et al. Hippocampal neurogenesis and antidepressive therapy: shocking relations. Neural Plast 2014;2014:723915.

38. Wachtel LE, Reti IM, Dhossche DM, Slomine BS, Sanz J. Stability of neuropsychological testing during two years of maintenance electroconvulsive therapy in an autistic man. Prog Neuropsychopharmacol Biol Psychiatry 2011;35:301-302.

39. Fisher P. Psychological factors related to the experience of and reaction to electroconvulsive therapy. J Ment Health 2012;21:589599.

40. Philpot M, Treloar A, Gormley N, Gustafson L. Barriers to the use of electroconvulsive therapy in the elderly: a European survey. Eur Psychiatry 2002;17:41-45

41. Dodero L, Damiano M, Galbusera A, et al. Neuroimaging evidence of major morpho-anatomical and functional abnormalities in the BTBR T + TF/J mouse model of autism. PLoS One 2013;8:e76655.

42. Wahlsten D, Metten P, Crabbe JC. Survey of 21 inbred mouse strains in two laboratories reveals that BTBR T/+ tf/tf has severely reduced hippocampal commissure and absent corpus callosum. Brain Res 2003;971:47-54.

43. Paul LK, Brown WS, Adolphs R, et al. Agenesis of the corpus callosum: genetic, developmental and functional aspects of connectivity. Nat Rev Neurosci 2007;8:287-299.

44. Badaruddin DH, Andrews GL, Bolte S, et al. Social and behavioral problems of children with agenesis of the corpus callosum. Child Psychiatry Hum Dev 2007;38:287-302.
45. Lau YC, Hinkley LB, Bukshpun P, et al. Autism traits in individuals with agenesis of the corpus callosum. J Autism Dev Disord 2013;43:1106-1118.

46. Yang M, Clarke AM, Crawley JN. Postnatal lesion evidence against a primary role for the corpus callosum in mouse sociability. Eur J Neurosci 2009;29:1663-1677.

47. Zikopoulos B, Barbas H. Altered neural connectivity in excitatory and inhibitory cortical circuits in autism. Front Hum Neurosci 2013;7:609.

48. Meziane H, Schaller F, Bauer S, et al. An early postnatal oxytocin treatment prevents social and learning deficits in adult mice deficient for Magel2, a gene involved in Prader-Willi syndrome and autism. Biol Psychiatry 2014.

49. Riddle WJ, Scott AI, Bennie J, Carroll S, Fink G. Current intensity and oxytocin release after electroconvulsive therapy. Biol Psychiatry 1993;33:839-841.

50. Smith JE, Williams K, Burkett S, Glue P, Nutt DJ. Oxytocin and vasopressin responses to ECT. Psychiatry Res 1990;32:201-202.

51. Devanand DP, Lisanby S, Lo ES, et al. Effects of electroconvulsive therapy on plasma vasopressin and oxytocin. Biol Psychiatry 1998:44:610-616.

52. Silverman JL, Yang M, Turner SM, et al. Low stress reactivity and neuroendocrine factors in the BTBR T $+\mathrm{tf} / \mathrm{J}$ mouse model of autism. Neuroscience 2010;171:1197-1208.

53. Bales KL, Solomon M, Jacob S, et al. Long-term exposure to intranasal oxytocin in a mouse autism model. Transl Psychiatry 2014;4: e480.

54. Hollander E, Novotny S, Hanratty M, et al. Oxytocin infusion reduces repetitive behaviors in adults with autistic and Asperger's disorders. Neuropsychopharmacology 2003;28: 193-198.

55. Miller M, Bales KL, Taylor SL, et al. Oxytocin and vasopressin in children and adolescents with autism spectrum disorders: sex differences and associations with symptoms. Autism Res 2013;6:91102.

56. Kalueff AV, Tuohimaa P. Contrasting grooming phenotypes in three mouse strains markedly different in anxiety and activity (129S1, BALB/c and NMRI). Behav Brain Res 2005;160:1-10.

57. Calcagnoli F, de Boer SF, Althaus M, den Boer JA, Koolhaas JM. Antiaggressive activity of central oxytocin in male rats. Psychopharmacology (Berl) 2013;229:639-651.

58. Sala M, Braida D, Lentini D, et al. Pharmacologic rescue of impaired cognitive flexibility, social deficits, increased aggression, and seizure susceptibility in oxytocin receptor null mice: a neurobehavioral model of autism. Biol Psychiatry 2011;69:875-882.

59. Francis SM, Sagar A, Levin-Decanini T, et al. Oxytocin and vasopressin systems in genetic syndromes and neurodevelopmental disorders. Brain Res 2014;1580:199-218. 